\title{
The relationship between talin and acetylcholine receptor clusters in
}

\section{Xenopus muscle cells}

\author{
M. WILLIAM ROCHLIN, QIMING CHEN, MARKUS TOBLER*, CHRISTOPHER E. TURNER, \\ KEITH BURRIDGE and H. BENJAMIN PENG†
}

Department of Cell Biology and Anatomy, Laboratories for Cell Biology, University of North Carolina, CB 7090, 108 Sruing Building, Chapel Hill, NC 27599, USA

* Present address: Universitaetsspital Zurich, Zurich, Switzerland

$\dagger$ Author for correspondence

\section{Summary}

Talin is involved in mediating the cytoskeleton-extracellular matrix interaction at focal contacts in cultured fibroblasts. Recently this protein has been localized at both the myotendinous junction (MTJ) and the neuromuscular junction (NMJ) in skeletal muscle. At the MTJ, talin may mediate the insertion of myofibrils into the plasma membrane, thus serving a function similar to that seen at focal contacts. However, the function of talin at the NMJ is unknown. In this study, we examined its distribution at both mature and developing acetylcholine receptor $(\mathrm{AChR})$ clusters in Xenopus muscle cells both in vivo and in vitro with immunofluorescence. At the NMJs of both myotomal and submaxillaris muscles, talin was absent from the AChR clusters. In cultured myotomal muscle cells, it was absent from $40 \%$ of both the nerve-associated AChR patches and the spontaneously formed $\mathrm{AChR}$ clusters located on the top surface of the cells. We therefore conclude that this protein is not essential for maintenance of $\mathrm{AChR}$ clusters at the NMJ. In

addition to MTJs, talin was invariably associated with AChR clusters induced by polyornithinecoated beads, and, to a large extent, with spontaneously formed clusters on the ventral side of cultured cells. A common feature of these talinpositive domains is the deep membrane infoldings, where bundles of actin filaments are inserted into the membrane. Thus, talin may be involved in the formation and maintenance of these structures. The deep membrane infoldings, though prominent at most NMJs, are absent from the two muscles under study in vivo. Our work thus suggests that the postjunctional membrane at the NMJ is heterogeneous, consisting of an AChR cluster domain and, often but not always, a domain for proteins involved in cytoskeleton-membrane linkage as exemplified by talin.

Key words: talin, neuromuscular junction, myotendinous junction, acetylcholine receptor.

\section{Introduction}

A key event in the development of the neuromuscular junction (NMJ) in skeletal muscle is the clustering of acetylcholine receptors (AChRs) in the postsynaptic membrane. The concentration of AChRs at sites of nerve-muscle contact is necessary for the muscle to respond appropriately to the release of neurotransmitter. Although a great deal is known about the timing of the clustering during development of the NMJ both in vivo (Chow \& Cohen, 1983) and in vitro (Anderson \& Cohen, 1977), much less is known about the mechanism of clustering. Neither the signal used by the nerve to initiate the clustering process, nor the molecules in the muscle cell that participate in this process, have been identified.
Although the NMJ is a cell-cell contact site, there are a number of reasons to suspect that cell-extracellular matrix interactions play an important role in inducing and maintaining the AChR clusters: (1) several extracellular matrix molecules, such as a heparan sulphate proteoglycan and $\mathrm{J} 1$, are colocalized with the $\mathrm{ACh} R$ clusters at the NMJ (Anderson \& Fambrough, 1983; Sanes et al. 1986). In fact, an initial step in the formation of NMJs involves the breakdown of the extracellular matrix followed by a new deposition of this material from muscle at the site of AChR clustering (Anderson, 1986). (2) The junctional basal lamina of frog skeletal muscle is capable of directing the formation of $\mathrm{AChR}$ clusters in the absence of nerve in vivo (Burden et al. 1979). Furthermore, agrin, a molecule extracted from the extracellular 
matrix of AChR-rich Torpedo electric organ, can direct the formation of $\mathrm{AChR}$ clusters in cultured muscle cells (Nitkin et al. 1987). (3) Polycation-coated beads, which presumably mimic the local cell-substrate interaction at nerve-muscle contacts, are capable of directing the formation of AChR clusters in vitro (Peng, 1987). Clearly, the relationship between cell-substrateassociated molecules and AChR clustering deserves further study.

Talin is a $225 \times 10^{3} M_{\mathrm{r}}$ protein which is thought to be involved in fibroblast focal adhesion formation and stabilization (Burridge \& Connell, 1983). It binds to both integrin and vinculin at these sites and may therefore link extracellular matrix molecules with cytoskeletal molecules (Buck \& Horwitz, 1987; Burridge, 1986). Immunohistochemical studies have shown that talin is colocalized with AChR clusters at rat NMJs (Sealock et al. 1986) and is also present at the myotendinous junction (MTJ) in chick (Tidball et al. 1986). The function of talin at these sites is not known. In this study, we have examined the relationship between talin and developing AChR clusters in cultured Xenopus muscle cells and compared this with the distribution of these proteins in vivo. Since the MTJ is a site at which the muscle fibre interacts with extracellular matrix molecules (Trotter et al. 1981), and is a membrane domain that is distinct from AChR cluster domains, we compared the talin staining of the NMJ and the MTJ. Although the MTJ does not form in vitro, both polycation-coated beads and the coverslip form tight associations with the muscle surface (Bloch \& Geiger, 1980; Moody-Corbett \& Cohen, 1982; Peng \& Cheng, 1982), which may resemble the MTJ but are coincident with AChR clusters. The talin distribution at these cell-substrate sites is quantified for comparison with that at the in vitro NMJs. In this paper, we present evidence to show that, whereas talin is always associated with the MTJ, its presence at the NMJ is highly variable. This variability suggests that talin is not involved in the maintenance of the clusters, although it may be involved in the assembly of specializations present at the clusters. (This work was previously communicated in an abstract form (Peng et al. 1987).)

\section{Materials and methods}

\section{Immunoblot and protein overlay analysis}

Tadpole tail muscle homogenate was subjected to electrophoresis on $10 \%$ SDS-polyacrylamide gels (Laemmli, 1970) containing $0.13 \%$ bisacrylamide prior to overnight electrophoretic transfer of the resolved proteins onto nitrocellulose membrane (Towbin et al. 1979). Following blocking of unoccupied protein binding sites with $3 \%$ bovine serum albumin (BSA), $0.05 \%$ Tween-20, $0.2 \%$ gelatin in Tris-buffered saline (TBS: $50 \mathrm{~mm}$ Tris- $\mathrm{HCl}$, pH $7.6,150 \mathrm{~mm}-\mathrm{NaCl}, 0.1 \% \mathrm{NaN}_{3}$ ) for $120 \mathrm{~min}$, the nitrocellulose strips were processed for immunoblotting or protein overlay analysis. For immunoblot analysis the strips were incubated at room temperature for $90 \mathrm{~min}$ with antichicken gizzard smooth muscle talin antiserum (Burridge $\&$ Connell, 1983) diluted 1:1000 in blocking buffer. Following extensive washing in blocking buffer minus BSA the nitrocellulose strips were incubated for $60 \mathrm{~min}$ at room temperature with radio-iodinated (Hunter \& Greenwood, 1962), affinity-purified goat anti-rabbit $\operatorname{lgG}\left(5 \times 10^{5} \mathrm{cts} \mathrm{min}^{-1} \mathrm{ml}^{-1}\right)$. After a final wash, the nitrocellulose strips were air dried and exposed to $X$ ray film with an intensifying screen at $-70^{\circ} \mathrm{C}$.

For protein overlay analysis (Burridge \& Mangeat, 1984) vinculin was purified from chicken gizzard smooth muscle (Feramisco \& Burridge, 1980) and ${ }^{125}$ I-labelled using the iodogen method (Fraker \& Speck, 1978). The blocked nitrocellulose strips were incubated with $\left[{ }^{125} \mathrm{I}\right]$ vinculin at $10^{6} \mathrm{cts} \mathrm{min}^{-1} \mathrm{ml}^{-1}$ diluted in $2 \%$ haemoglobin, $0.2 \%$ gelatin, $0.05 \%$ Tween-20, and TBS, $\mathrm{pH} 7.6$, for $90 \mathrm{~min}$ at room temperature. The strips were subsequently washed, air-dried, and exposed to $\mathrm{X}$-ray film as described for the immunoblotting.

\section{Muscle cell culture and bead treatment}

The techniques for preparing nerve and muscle cell cultures from Xenopus laevis embryos followed those described by Peng \& Nakajima (1978). The muscle cells were plated directly on no. 1 coverglass squares, but the nerve-muscle cocultures were plated on collagen-coated coverglasses. For nerve-muscle cocultures, culture medium was supplemented with $6 \mathrm{~mm}$ $\mathrm{CaCl}_{2}$ to increase the number of NMJs (Henderson et al. 1984). Treatment of muscle cells with polyornithine-coated latex beads also followed previously published methods (Peng \& Cheng, 1982).

\section{Immunofluorescence}

After 22-36h, cultures were labelled with tetramethylrhodamine-conjugated $\alpha$-bungarotoxin (R-BTX) (Molecular Probes, Eugene, OR), fixed in Luther's fixative (containing $80 \mathrm{~mm}$ cyclohexylamine, $0.5 \%$ paraformaldehyde in $40 \mathrm{~mm}$-Pipes buffer, pH 6.5) for $30 \mathrm{~min}$ (Luther $\&$ Bloch, unpublished data), and washed with a buffer containing $10 \mathrm{mM}-\mathrm{MgCl}_{2}, 10 \mathrm{~mm}-$ $\mathrm{NaN}_{3}, 0.5 \%$ Brij 58 (Sigma) and $75 \mathrm{~mm}$-Tris- $\mathrm{HCl}(\mathrm{pH} 6.7)$. Non-specific binding sites were blocked in $20 \mathrm{~mm}$-Tris- $\mathrm{HCl}$ buffer containing $0 \cdot 1 \%$ BSA. The cultures were then treated with rabbit anti-talin IgG overnight at $4^{\circ} \mathrm{C}$. Following extensive washing, they were treated with FITC-conjugated goat antirabbit IgG (Organon Technika, Cochranville, PA) overnight for 2 days at $4^{\circ} \mathrm{C}$. After thorough washing, the culture coverglass was mounted on a slide. Microscopy was performed using a Leitz Orthoplan microscope equipped with fluorescence optics (E. Leitz, Inc., Rockleigh, NJ). Photographs were taken with an Olympus OM-2 camera using Kodak Tri-X film.

\section{Preparation of larval muscle fibres}

Tadpoles were anaesthetized with $0.1 \%$ tricaine methanesulphonate (MS-222). The tail was skinned and treated with $3 \mathrm{mg} \mathrm{ml}^{-1}$ collagenase (type 1 from Sigma) in frog Ringer solution for $2-3 \mathrm{~h}$. With moderate agitation, individual muscle fibres could be dissociated. They were collected by gentle centrifugation and fixed in Luther's fixative. After washing, they were labelled with R-B TX, permeabilized, and reacted with antibodies in the same manner as the tissue cultures.

In addition to dissociated myotomal muscle fibres of the tail, we also examined whole mounts of the submaxillaris muscle After removal from the tadpole, it was labelled with R-BTX prior to fixation, but otherwise the procedure for labelling whole mount and dissociated cells was the same. After labelling of this muscle was complete, the connective and cartilaginous tissues were pared away, and the muscle was mounted and examined as described for the tissue cultures.

\section{Electron microscopy}

Cultures were prepared in Permanox $35 \mathrm{~mm}$ dishes (Miles Scientific, Lisle, IL). They were fixed with $1 \%$ glutaraldehyde in $0.05 \mathrm{M}$-sodium cacodylate buffer $(\mathrm{pH} 7.2)$ for $2 \mathrm{~h}$, washed, 
and postfixed with $1 \% \mathrm{OsO}_{4}$ for $1 \mathrm{~h}$. They were then stained $e n$ bloc with $1 \%$ uranyl acetate in $50 \mathrm{~mm}$-acetate buffer $(\mathrm{pH} 5.5)$ for $2 \mathrm{~h}$, dehydrated through an ethanol series, and embedded in Epon. After the block was removed from the dish, single cells were cut out with a jeweler's saw and mounted on blank Epon blocks. Thin sections were cut perpendicular to the original culture substratum, stained with uranyl acetate and lead citrate, and examined with a JEOL 100CX electron microscope. Larval tail muscle was prepared for electron microscopy in a similar way.

For quantification of electron micrographs, a Hipad digitizer (Houston Instruments, Austin, TX) interfaced with an IBM PC-AT computer was used to measure the length of membrane profiles.

\section{Results}

\section{Immunoblots}

Immunoblot analysis demonstrated that the anti-talin antibody recognized a protein band of $225 \times 10^{3} M_{\mathrm{r}}$ in the tadpole tail homogenate (Fig. 1, lane 3). The antibody

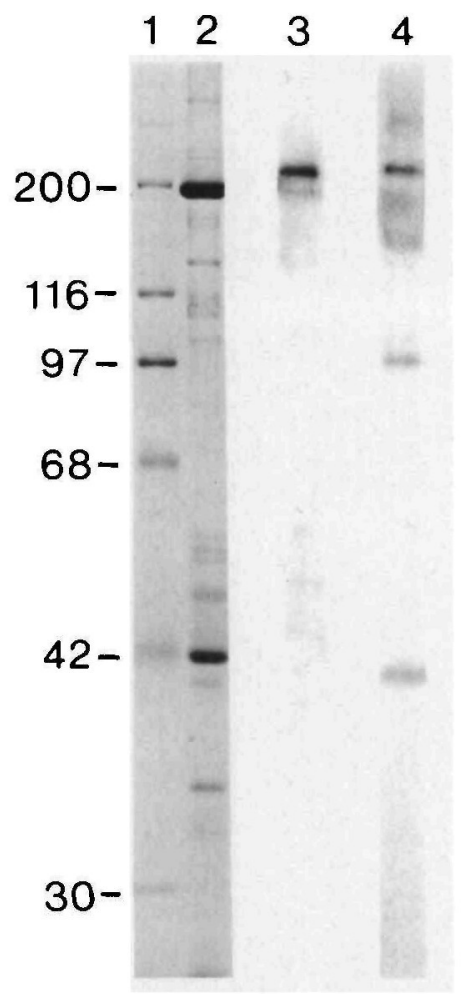

Fig. 1. Presence of talin in tadpole tail musculature. Homogenates were prepared from stage 54 tadpole tails and electrophoresed on SDS-PAGE gels as described in Materials and methods. Lanes 1 and 2 are Coomassie Blue-stained gels containing molecular weight markers $\left(\times 10^{3}\right)$ and tail homogenate, respectively. Lane 3 is an autoradiograph of a nitrocellulose blot after labelling with anti-talin and ${ }^{125} \mathrm{I}$ conjugated secondary antibody. The two labelled bands are of molecular weight 225 and $190\left(\times 10^{3}\right)$, corresponding to talin and its major proteolytic fragment. Lane 4 is an autoradiograph following a vinculin overlay assay. The nitrocellulose blot was incubated with ${ }^{125}$ I-labelled vinculin. In addition to labelling talin, weaker labelling of $\alpha$-actinin was also observed. also weakly labelled a $190 \times 10^{3} M_{\mathrm{r}}$ band, which corresponds to the proteolytic fragment of talin as reported (O'Halloran \& Burridge, 1986). Further evidence that the $225 \times 10^{3} M_{\mathrm{r}}$ band was indeed talin was obtained by overlaying a similar blot of the tadpole tail homogenate with ${ }^{125} \mathrm{I}$-labelled vinculin, a protein that binds talin (Burride \& Mangeat, 1984). As shown in Fig. 1 (lane 4), this probe predominantly recognized the $225 \times 10^{3} \mathrm{M}_{\mathrm{r}}$ band. It also labelled $\alpha$-actinin $\left(100 \times 10^{3} M_{\mathrm{r}}\right)$, as has been demonstrated (Burridge \& Mangeat, 1984; Wachsstock et al. 1987; Wilkins et al. 1983). These data demonstrate that a molecule homologous to chicken gizzard talin is present in the myotomes of the tadpole tail.

\section{Talin at NMY and MTY in vivo}

Myotomal muscle fibres are innervated at the ends where MTJs also exist (Chow \& Cohen, 1983). Thus, NMJ and MTJ are in close proximity to each other (Fig. 10). When dissociated myotomal muscle fibres were stained with anti-talin, the ends of the fibres clearly showed the presence of this antigen (Fig. 2A). The talin-positive structures appeared as streaks covering both ends of the muscle fibre. These streaks correspond to the deep membrane invaginations of the MTJ, where the myofibrils make their insertion into the membrane (Fig. 10; cf. Tidball et al. 1986). In such side views, R-BTX staining often overlapped with the antibody staining, thus giving a false impression of colocalization (data not shown). However, when the ends of the fibres were viewed end-on (shown in Fig. 2C-D) the AChR clusters were observed to reside in membrane domains (Fig. 2C) that were deficient in talin (Fig. 2D). This conclusion was based on data gathered from more than 200 muscle fibres. The talin staining was usually more extensive than the AChR staining. In fact, a small fraction of the muscle fibres were innervated only at one end, but talin was still associated with the MTJs at both ends (data not shown).

Further support for these observations was obtained by examining the NMJ and MTJ of the submaxillaris muscle. In Xenopus tadpoles, this muscle is very thin and can be viewed with high resolution in whole mounts. Unlike the myotomal muscle in the tail, the NMJ forms away from the ends of the fibres, and therefore away from the MTJ in the submaxillaris. Double staining of this muscle with R-BTX and antibody demonstrated clearly that only the MTJs of the fibres had an enrichment of talin above the background and not the NMJs (Fig. 2E-F). Thus, in both submaxillaris and myotomal muscle, AChR clusters at the NMJ were devoid of talin.

These results vary from the colocalization of talin with the NMJ in rat diaphragm muscle (Sealock et al. 1986). This variation may be due to the fact that the NMJs in these larval muscles lack deep membrane invaginations (see Fig. 10A, below). Thus, we examined cryosections of Xenopus leg muscles, which exhibit postjunctional infoldings as in rat diaphragm. As shown in Fig. 3, the NMJs identified by R-BTX staining were indeed associated with talin immunoreactivity. However, because of the thickness of these sections $(4 \mu \mathrm{m})$, it is not possible to assess the relationship between talin and the membrane 
domain occupied by $\mathrm{AChR}$ clusters in the postsynaptic membrane.

No staining of the MTJ of myotomal muscle fibres was found if the preimmune serum (Fig. 2B) was used in place of the anti-talin antibody.

Talin at nerve-associated receptor patches (NARPs) in vitro

To investigate the association of talin with developing NMJs, we examined the AChR clusters in 2- to 3-day-old nerve-muscle cocultures. In cultured muscle cells, talin was present both diffusely as well as in concentrated patches, as Fig. 4 (middle column) shows. In this study, we focussed on the relationship between NARPs and these concentrated talin patches. The presence of talin patches was highly variable at NARPs. We categorized this variation into three classes, as shown in Fig. 4. In the first class $(4 \mathrm{~A}-\mathrm{C})$, the NARP was extensively (close to $100 \%$ of its length) associated with talin. In Fig. 4C, a nerve that induced such a talin-positive $\mathrm{NMJ}$ can be seen coursing over the muscle cell. Fig. $4 \mathrm{~A}$ displays the RBTX staining. As was common in these cocultures, the NARPs were not continuous along the entire path of apparent nerve-muscle contact. All the NARPs in this example were positive in talin, but not all talin accumulations were sites of $\mathrm{AChR}$ clusters (compare Fig. $4 \mathrm{~B}$ and A). In the second class, less than $50 \%$ of the extent of RBTX staining was positive for talin as shown in Fig. 4D-F. In this example, NARPs located within segments 1 and 3 (Fig. 4D) were talin-positive (Fig. 4E),
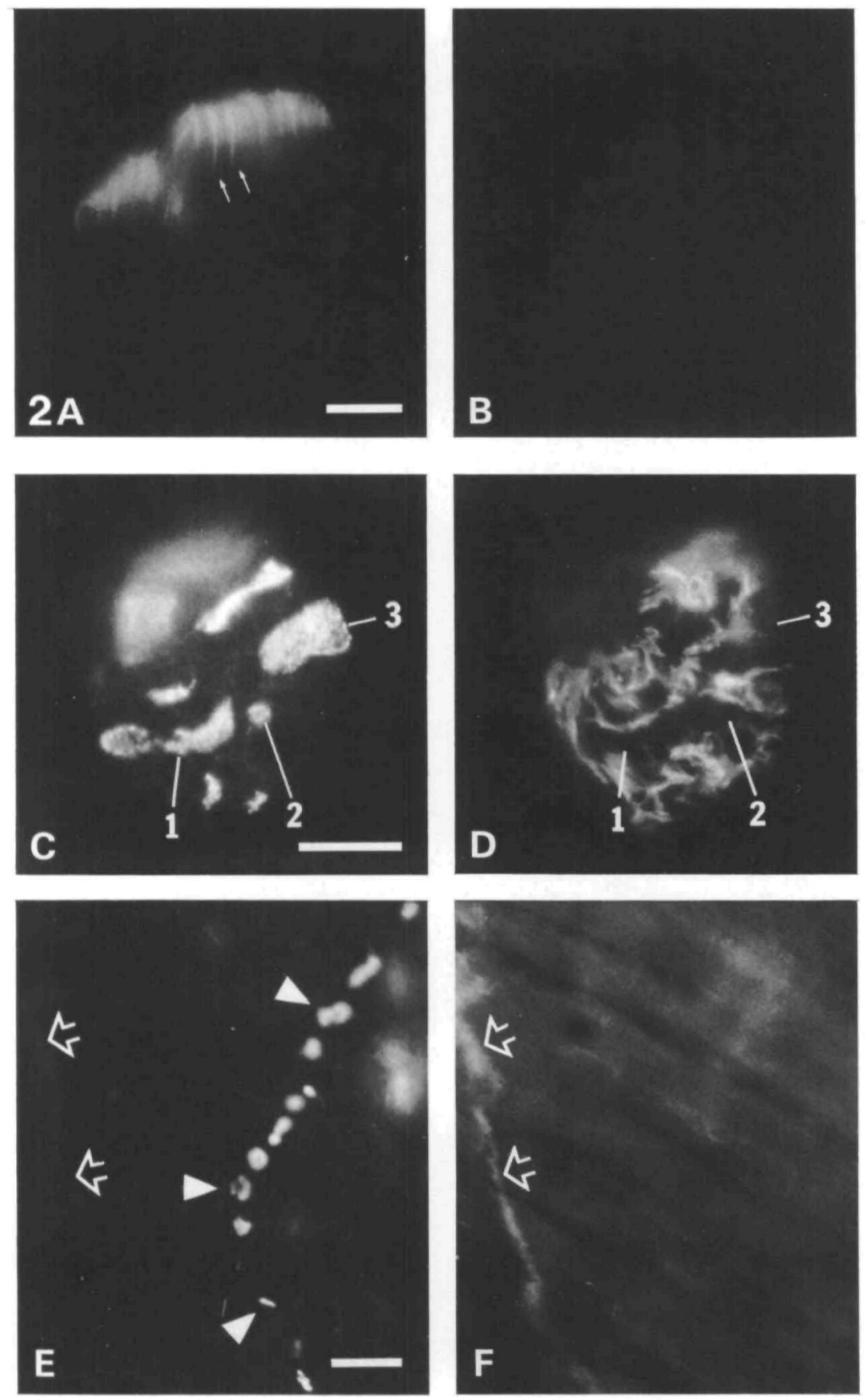

Fig. 2. Talin in muscle fibres in vizo. A. Antitalin staining of an isolated myotomal muscle fibre obtained from a stage 47 tadpole. The fibre extends downward from the intensely labelled MTJ. The arrows point to talinpositive streaks, which correspond to deep infoldings at this MTJ. B. A similar region of a fibre labelled with pre-immune serum. C-D. An example of myotomal muscle fibres doubly labelled with R-BTX (C) and anti-talin (D). The end of this fibre faced the coverslip, affording an end-on view of the MTJ and NMJ. The receptor patches (labelled $1-3$ in C) were predominantly localized in talin-negative areas (similarly labelled in D). In all, more than 200 muscle fibres were subject to this type of analysis with consistent results. E-F. A whole-mounted submaxillaris muscle from a stage 48 tadpole was double labelled with $R$ BTX (E) and anti-talin (F). Filled arrowheads in $\mathrm{E}$ indicate $\mathrm{AChR}$ clusters at NMJs of a series of muscle fibres. Open arrowheads in both $E$ and $F$ point to the MTJ. Talin was not elevated at the NMJ (E), but was elevated at the MTJ of this muscle (F). Bars: A-D, $10 \mu \mathrm{m} ; \mathrm{E}, \mathrm{F}$, $20 \mu \mathrm{m}$. 

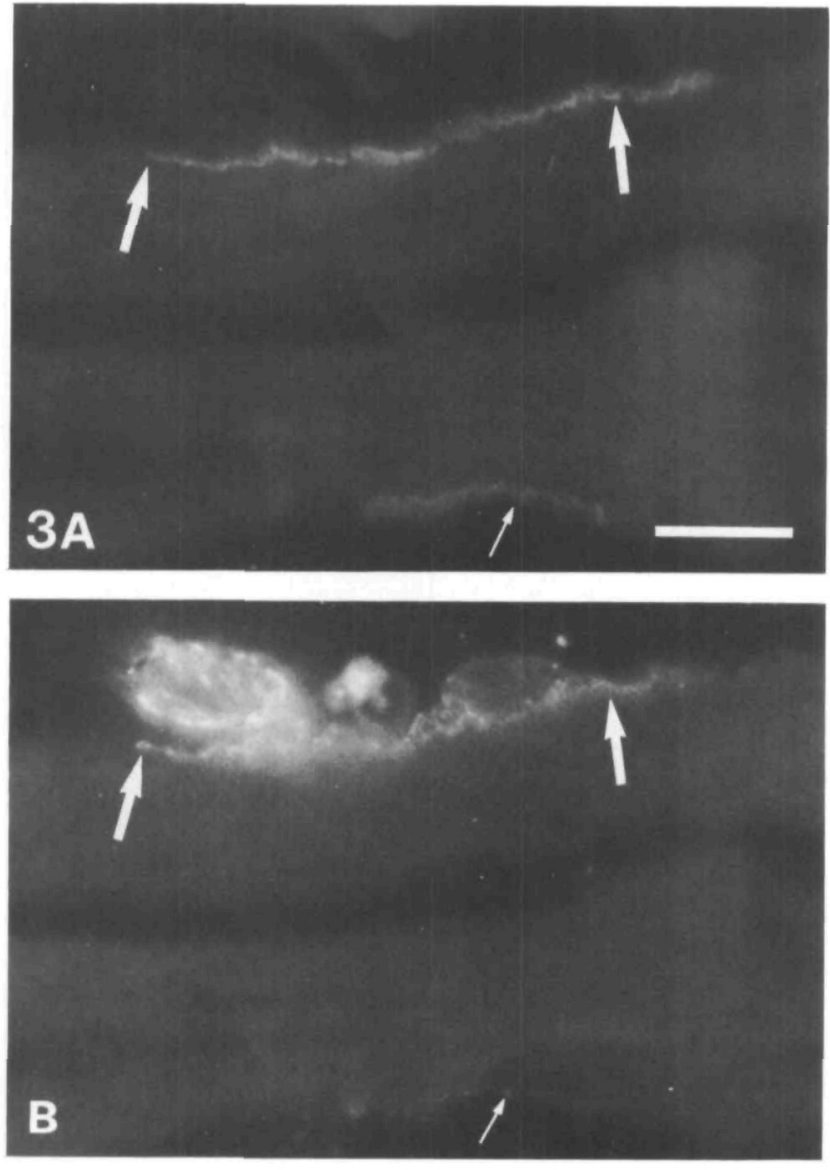

Fig. 3. Talin at Xenopus leg muscle NMJ. A. R-BTX staining; $\mathrm{B}$, the corresponding anti-talin staining. Two longitudinally sectioned muscle fibres and portions of their NMJs are evident in A. Both NMJs were also sites of increased talin staining. An unidentified cell overlying the upper muscle fibre was also intensely stained for talin. Close examination reveals that the staining patterns were not identical (e.g. as indicated by the arrows). Bar, $10 \mu \mathrm{m}$.

whereas those located within segment 2 were not. In the third class, talin staining was not intensified over the diffuse staining level at NARPs along the entire length of the nerve-muscle contacts (Fig. 4G-I). In this example, the contact area occupied by NARPs (between filled arrowheads in Fig. 4G) did not show antibody staining above the diffuse staining of the cell, whereas a R-BTXnegative area along the opposite edge of the same cell (between open arrowheads in Fig. 4H) clearly showed a much elevated level of staining.

These three classes were quantified in Fig. 5A. Approximately $40 \%$ of the NARP-positive nerve-muscle contacts had extensive talin association, $15 \%$ had incomplete association, and $45 \%$ were talin-negative. These data suggest that, unlike the endplates in vivo, $55 \%$ of the newly formed $A C h R$ clusters in vitro have an association with talin. To gain insight into the cause of this variability, we studied the talin distribution at both aneural, spontaneously formed clusters and at beadinduced clusters.
Talin at spontaneously occuming receptor patches (hotspots) in vitro

In the absence of innervation, AChR clusters are formed spontaneously on muscle cells (Anderson \& Cohen, 1977). The distribution of talin at these hotspots was evaluated. As with NARPs, we saw variability in the association of talin with hotspots. In Fig. 6A, a series of AChR clusters were located at the periphery of the cell in an outwardly radiating pattern. The talin staining (Fig. 6B) reveals that every cluster was in register with a talin patch (arrowheads in Fig. 6A and B). The pattern of these talin patches was reminiscent of that in fibroblasts, in which this protein is present at focal contacts (Burridge, 1986). Although all of these hotspots were talinpositive, not every talin patch was associated with a hotspot (thin arrows in Fig. 6A,B). Fig. 6C-D shows additional examples of a strong $\mathrm{ACh}$-talin association. The arrowheads point out a close matching in the pattern of R-BTX and talin staining. However, not all hotspots had a detectable talin concentration. For example, while hotspots 3 and 4 in Fig. $6 \mathrm{E}$ were clearly talin-positive, hotspots 1 and 2 were stained incompletely for talin. In addition, some hotspots, e.g. hotspot 5 in Fig. 6E, were totally devoid of talin. Thus, variability existed both within the same cell as well as between cells.

These observations are quantified in Fig. 5B. The association of talin with receptor clusters was categorized into three classes as exemplified in Fig. 6: positive, incomplete and negative association. Nearly $60 \%$ of the hotspots were positively stained with anti-talin. Approximately $21 \%$ of the hotspots were partially co-stained with anti-talin, and less than $20 \%$ were talin-free.

In non-muscle cells, focal contacts, areas of tight adhesion to the substratum, are enriched in talin (Burridge, 1986). Since it is known that hotspots that form on the ventral surface of muscle cells are usually the sites of cell-substrate contacts (Bloch \& Geiger, 1980), we predicted that hotspots on this surface would be more likely to colocalize with talin than those on the dorsal surface of the cell. As illustrated in Fig. 7, substratumassociated hotspots, as well as those associated with the edge of the muscle cells, were more frequently coextensive with talin than dorsal hotspots. Whereas more than $60 \%$ of the ventral and edge hotspots were intensely talin positive, only $30 \%$ of the dorsal hotspots fell into this category. Conversely, hotspots that were devoid of talin were more heavily represented on the top surface of the cell $(43 \%)$ than on the substratum surface $(11 \%)$ or along the edge $(19 \%)$. This variability could not be due to a difference in the ability of the ventral and dorsal surfaces to accumulate talin at AChR cluster sites, as indicated by the experiments described below.

\section{Talin at bead-associated receptor patches (BARPS)}

Basic polypeptide-coated latex beads provide us with a stimulus of receptor clustering that can be initiated at a controlled time (Peng $\&$ Cheng, 1982). It was therefore of interest to use this model system to probe the correspondence between talin and nascent $\mathrm{AChR}$ clusters and to examine the timing of the appearance of $\mathrm{AChRs}$ and talin. AChR clusters can be detected at the bead-muscle 

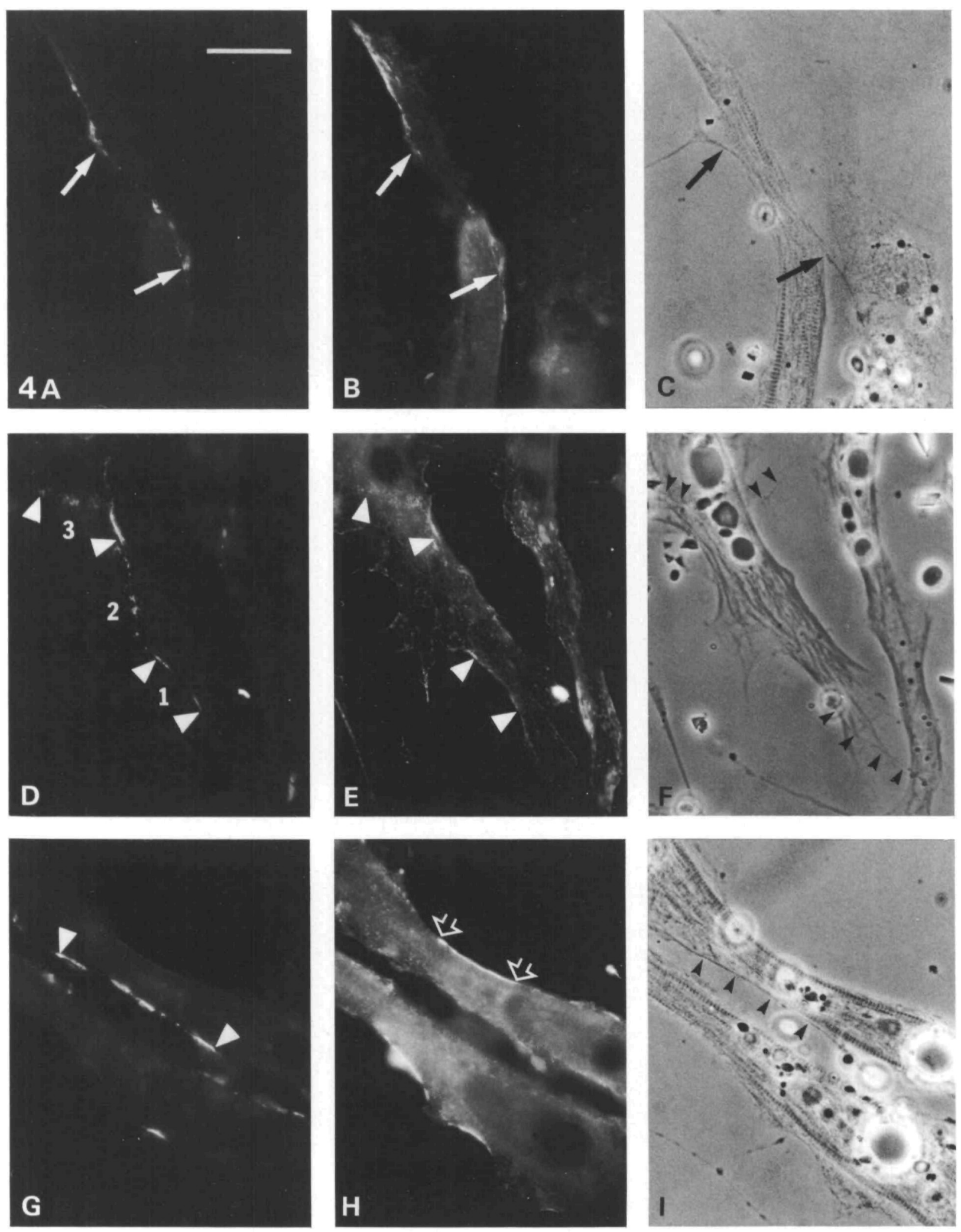

Fig. 4. Talin in NARPs. A,D,G. R-BTX staining; B,E,H, the corresponding anti-talin staining; C,F,I, the corresponding phase-contrast views. The top row is an example of a NARP that exhibited extensive colocalization with talin. The arrows mark the position of the NARP in A and B and the corresponding stretch of neurite in C. The middle row shows an example of a NARP with only intermittent anti-talin staining. Talin (E) was present at the segments of the NARP marked 1 and 3 (delimited by white arrowheads in $D$ and $E$ ) and absent from segment 2 . The bottom row provides an example of a talin-negative NARP (filled arrowheads in G). On the side of the cell opposite to the NARP, there was a talin accumulation (open arrowheads in $\mathrm{H}$ ). The arrowheads in F and I indicate the neurite. Bar, $20 \mu \mathrm{m}$. 

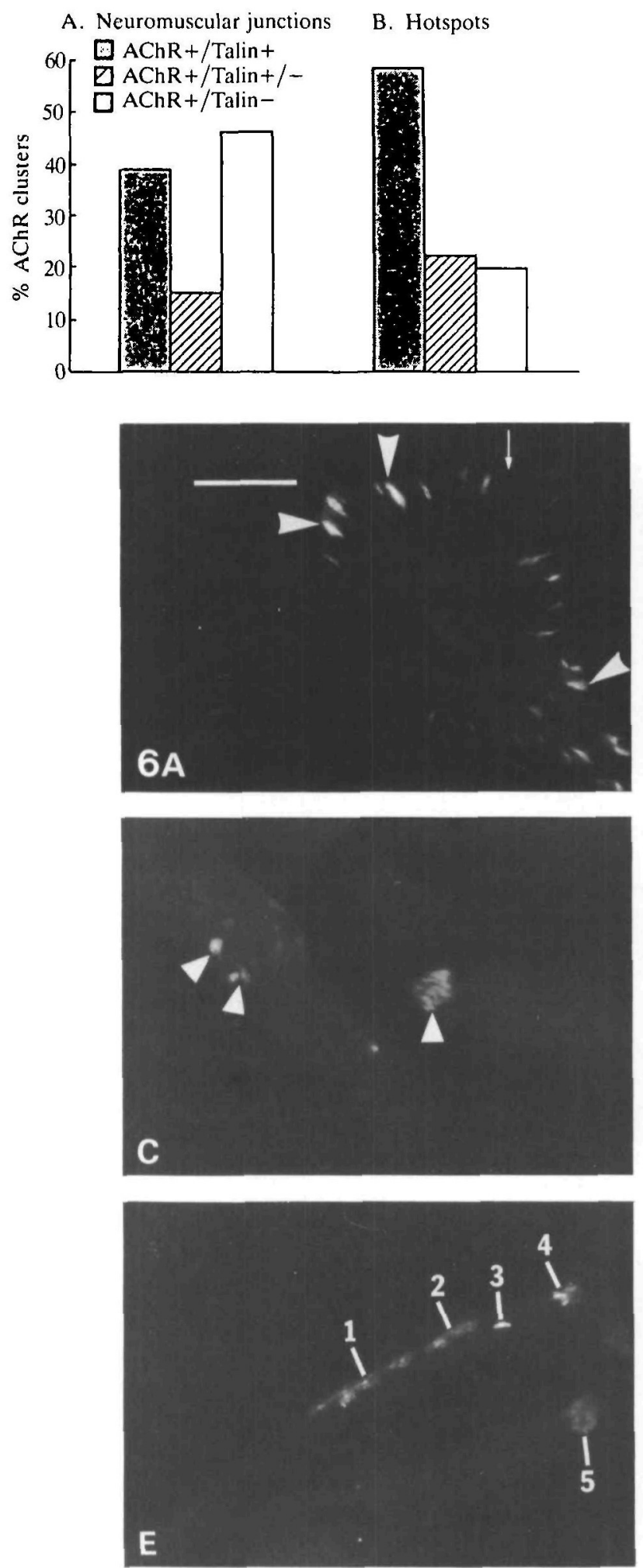

Fig. 5. Frequency histogram of the colocalization of receptor clusters and talin in cultured myotomal muscle cells. The ordinate represents the percentage of the total clusters that fell into the indicated categories. $\mathrm{ACh}+/$ Talin + : talin immunoreactivity coextensive with R-BTX staining; $\mathrm{AChR}+$ /Talin +/-: talin immunoreactivity present, but not coextensive with the R-BTX staining. AChR+/Talin - : no detectable talin staining. A. Colocalization of talin with NARPs; based on data collected from 123 nerve-muscle contacts in 12 separate experiments. B. Colocalization of talin with hotspots; based on data from $225 \mathrm{AChR}$ clusters in 67 cells in two separate experiments.
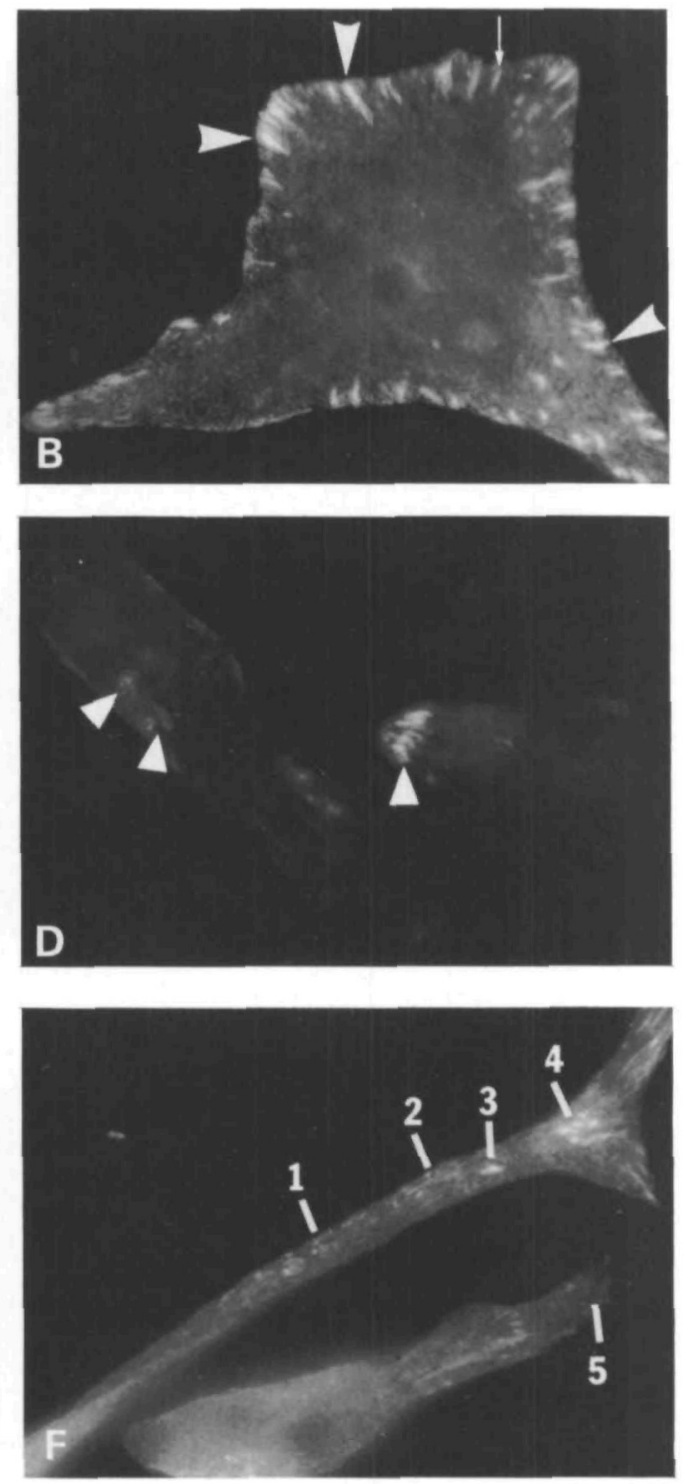

Fig. 6. Talin at hotspots. A,C,E. R-BTX staining; B,D,F, the corresponding anti-talin staining. A-D. Examples of a high degree of colocalization. Note that each hotspot in A colocalized with talin in B (arrowheads), but a number of talin patches were not sites of hotspots (e.g. thin arrow in B points to a talin-positive patch devoid of AChR clustering as shown in A). The muscle cells in $\mathrm{C}$ exhibited both small and large hotspots (arrowheads). As in the example above, these hotspots were extensively colocalized with talin (D). E-F. Example of a variable degree of colocalization. In E, a series of hotspots were present on the upper muscle cell. Hotspots 3 and 4 were intensely talin-positive, whereas hotspots 1 and 2 had little if any talin. Hotspot 5 , on the lower muscle cell appeared to be talin-negative (F). Thus, there was a high degree of variability in the presence and intensity of talin staining at hotspots both within the same cell and between cells. Bar, $20 \mu \mathrm{m}$. 


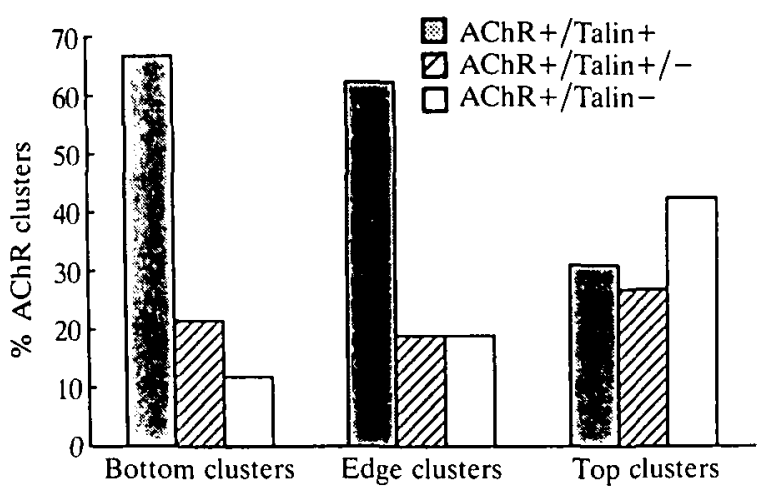

Fig. 7. Frequency histogram of talin-AChR cluster colocalization at ventral, edge and dorsal hotspots. The legend is defined in Fig. 5. Bottom and edge clusters had a high percentage of talin colocalization, whereas top clusters tended to be talin-negative; based on data from $151 \mathrm{AChR}$ clusters in 42 cells in two separate experiments.

contacts within 2-3 h of bead-muscle coculture (Peng \& Phelan, 1984). All of these early BARPs also demonstrated anti-talin staining (Fig. 8A-F). On the other hand, some of the talin-positive bead-muscle contacts had only weak to non-detectable R-BTX staining (Fig. 8A-C, bead-muscle contact no. 2). Note that not all of the beads that attach to the muscle cells induce accumulation of either of these markers (compare Fig. 8C with $\mathrm{A}$ and $\mathrm{B}$ ). However, of the beads that have induced either receptor clusters or talin accumulation, $45 \%$ were both $\mathrm{AChR}$ and talin-positive, and $55 \%$ were talinpositive but AChR-negative (Fig. 9).

By $7-8 \mathrm{~h}, 90 \%$ of the effective bead-muscle contacts were clearly both receptor and talin-positive, and only about $10 \%$ were receptor-negative (Fig. 8G-I and Fig. 9B). Both markers could be unambiguously detected at these sites, even though the fluorescence intensity was still relatively weak. After $24 \mathrm{~h}$ of bead-muscle incubation, the staining of both receptors (Fig. 8J) and talin (Fig. 8K) was intensified. Furthermore, virtually all beads that were positive for either of these markers were positive for both (Fig. 9C). These data indicate that talin arrived at effective bead-muscle contact sites prior to receptors and that both markers accumulated at these sites over a 24 -h period. Unlike NARPs and hotspots, there were no BARPs that were talin-negative.

\section{Ultrastructure of the NMY, the MTY and the BARP}

The high correspondence between talin and BARPs in vitro, and between talin and MTJs in vivo prompted us to compare the ultrastructures of these sites, and to contrast these with the ultrastructure of the NMJ. We chose to examine the NMJ and MTJ of the myotomal muscle fibres because we had studied the correlation between talin and AChR clusters in this muscle both in vivo and in vitro.

As noted before, the MTJ and the NMJ are located at the ends of myotomal muscle fibres in close apposition to each other. Fig. 10A shows the ultrastructures of these two types of specializations. The postsynaptic membrane, where AChRs are concentrated, showed a scal- loped appearance. Each protuberance (black arrowheads in Fig. 10A) was associated with a density and was separated from neighbouring protuberances by a shallow gutter composed of unspecialized membrane. At the MTJ, however, the membrane was deeply invaginated and myofibrils were inserted into the membrane at an oblique angle (white arrows in Fig. 10A). Thus, the presence of deep membrane invaginations is a feature of the MTJ, but not of the NMJ in the myotomal muscle fibres.

Previously, we showed that membrane invaginations are associated with BARPs (Peng \& Cheng, 1982). An example is shown in Fig. 10B. These deep invaginations are absent from the NMJ of the myotomal muscle fibres in vivo (Fig. 10) and from NARPs in vitro (Weldon \& Cohen, 1979). However, careful examination reveals that the invaginated membrane area at the BARP was often segregated into two distinct domains: a scalloped region proximal to the bead (black arrowheads in Fig. 10B), which resembled the postsynaptic membrane at the NMJ, and a more distal region, which received insertion of myofibrils (white arrows in Fig. 10B) comparable to the MTJ. Thus, these electron micrographs showed that the bead-induced ultrastructural specializations have features common to both the MTJ and the NMJ.

The onset of AChR clustering occurred rapidly following the bead-muscle contacts. Fig. 11 (triangles) shows the time course of the clustering process. Except for an earlier onset, the time course of the bead-talin association basically parallelled that of the AChRs (Fig. 9). The development of membrane invaginations at the bead-muscle contacts, as determined from electronmicroscopic images (Fig. 11, squares), had a much slower time course. This was determined by measuring the length of the membrane profile in excess of the length of the bead circumference at the bead-muscle contacts for different incubation periods up to $72 \mathrm{~h}$. Fig. 11 shows that the membrane invaginations continued to develop during this period. However, their appearance lagged considerably behind that of AChRs and talin: only a $10 \%$ increase in membrane length was seen by the time the number of $\mathrm{AChR}$ clusters already reached maximum. This suggests the bead-induced accumulation of talin and AChRs occurs prior to the development of the membrane invaginations. Whether talin appears at immature MTJs prior to infoldings in vivo is not known.

\section{Discussion}

Previous studies have demonstrated the presence of several cytoskeletal proteins (actin, vinculin, $\alpha$-actinin and talin) beneath the postsynaptic membrane of NMJs (Bloch \& Hall, 1983; Hall et al. 1981; Sealock et al. 1986). In other locations, the latter three proteins have been implicated in the attachment of bundles of actin filaments to the plasma membrane, for example, at fibroblast focal adhesions (Burridge, 1986) or at the myotendinous junction of skeletal muscle (Shear \& Bloch, 1985; Tidball et al. 1986; Trotter et al. 1983). We have studied the distribution of talin in Xenopus muscle 

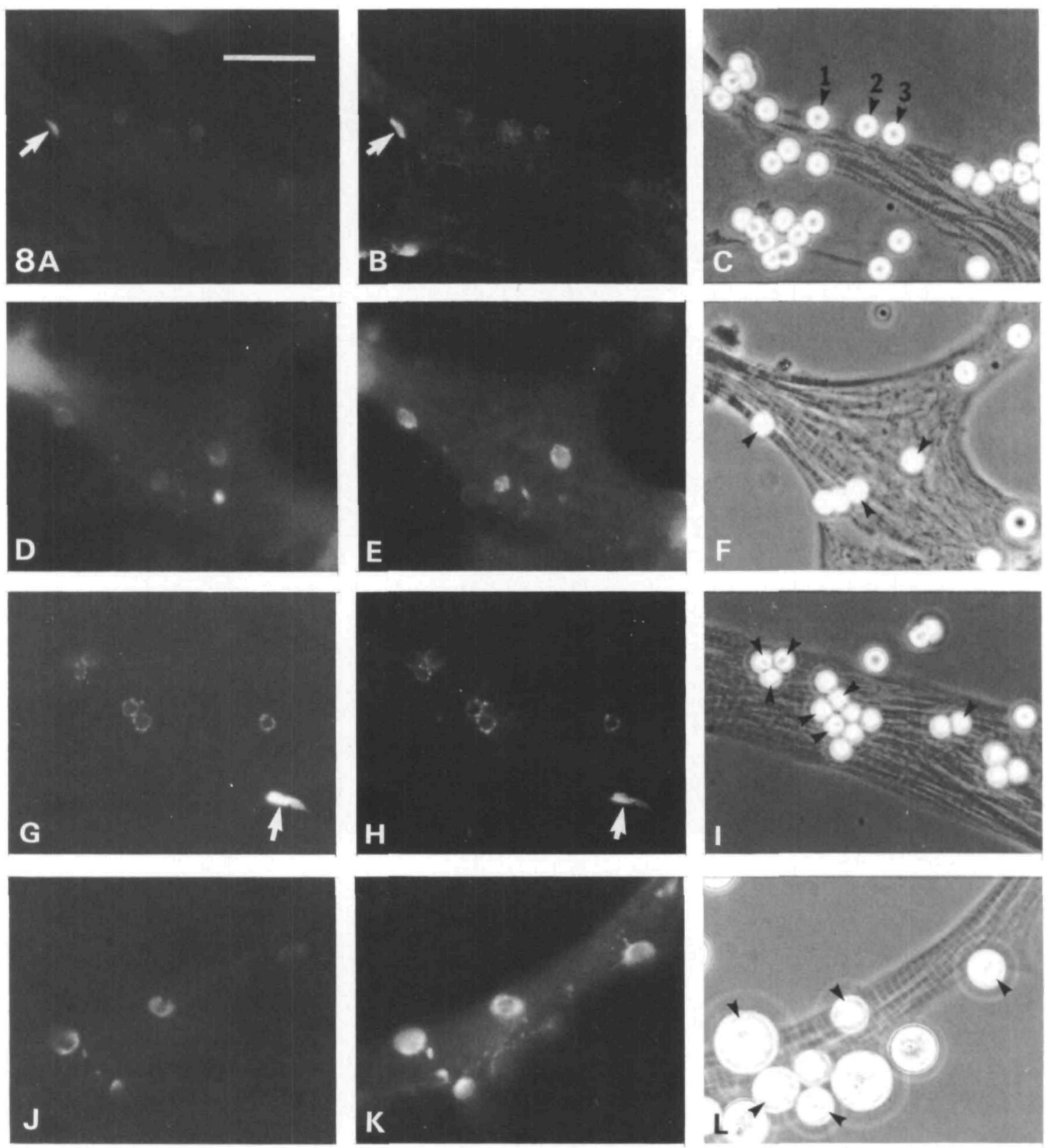

Fig. 8. Talin at BARPs. A,D,G,J. R-BTX staining; B,E,H,K, the corresponding anti-talin staining; C,F, I,L, the corresponding phase-contrast views. A-F. $2-3 \mathrm{~h}$ bead-muscle coculture with $4 \mu \mathrm{m}$ beads. Although talin accumulations were obvious at many bead-muscle contacts (arrowheads in C and F), the R-BTX staining was dim at all beads and absent from bead no. 2 in C. Note the talin-positive hotspot indicated by the arrow (A-B). G-I. 7-8h bead-muscle coculture. Nearly all of the beads (arrowheads in I) that had talin or a receptor cluster had both markers. A talin-positive hotspot was also present on this muscle cell (arrows). J-L. 22-24 h bead-muscle coculture. All beads (arrowheads in L) that had either a receptor cluster or talin had both markers. We used $9 \mu \mathrm{m}$ beads in this set of experiments. Bar, $20 \mu \mathrm{m}$.

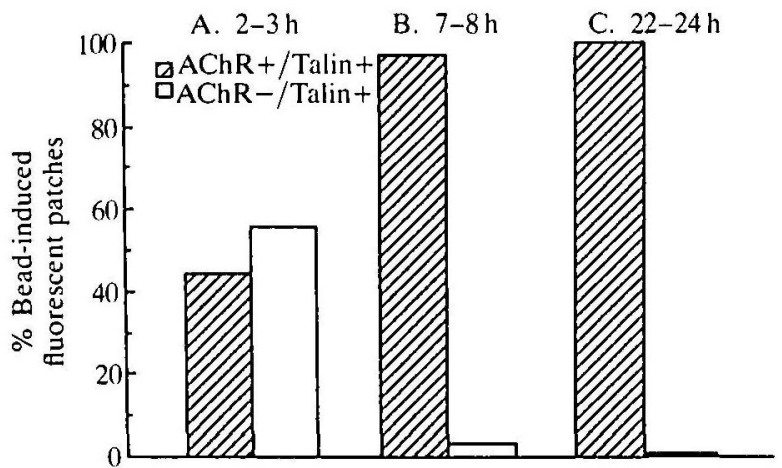

Fig. 9. Frequency histogram of talin-AChR cluster colocalization at the bead-muscle contacts. The number of bead-muscle contacts that were $\mathrm{AChR}+/ \mathrm{Talin}+$ or $\mathrm{AChR}-/$ Talin + were determined. They were expressed as . percentages of the sum of both groups (ordinate). There were no BARPs without talin staining. For each time period, a mean of 232 fluorescence-positive bead-muscle contacts were scored. 

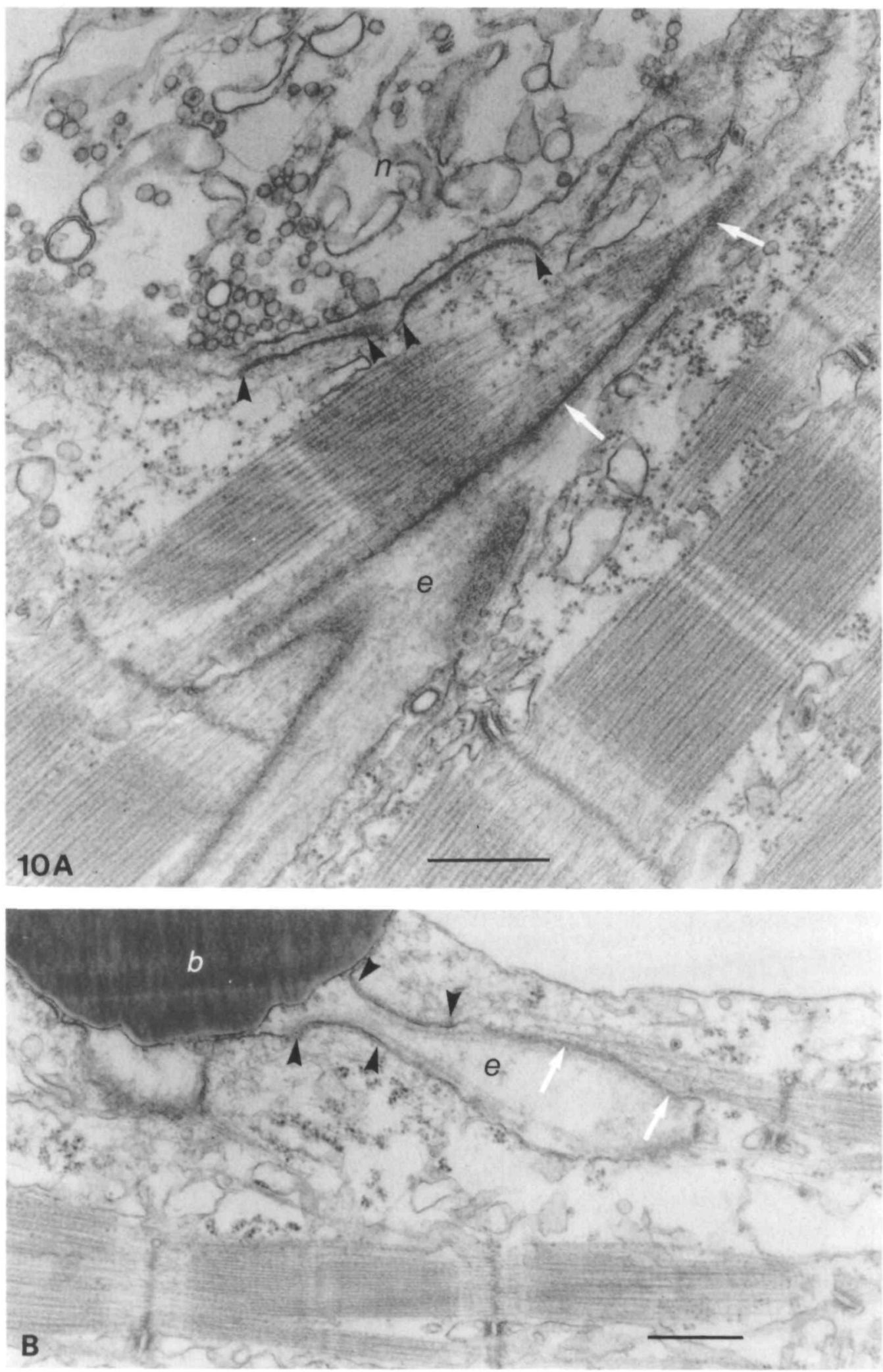

Fig. 10. Ultrastructures of the larval tail NMJ and MTJ (A) and of a bead-muscle contact on a cultured muscle cell (B). Both the NMJ and the bead-muscle contact exhibited membrane protuberances associated with a cytoplasmic density (black arrowheads), which presumably correspond to sites of $\mathrm{AChR}$ clusters. In the vicinity of the NMJ, one can see the deep membrane invaginations of the MTJ where myofibrils insert into the plasma membrane (white arrows in A). Similar invaginations at the bead-muscle contact also appear to be the site of myofibril insertion (white arrows in $B$ ). $e$, the extracellular space in the infoldings; $n$, nerve terminal; $b$, bead. Bar, $0 \cdot 5 \mu \mathrm{m}$. 


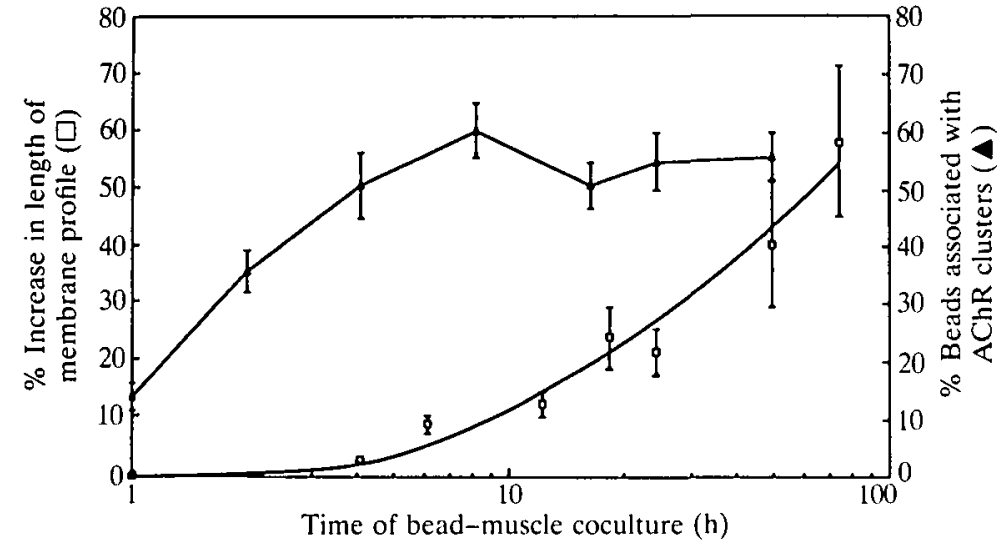

Fig. 11. Development of membrane infoldings and $A C h R$ clusters at bead-muscle contacts. BARP formation $(\boldsymbol{\Delta})$ occurred rapidly, with nearly $40 \%$ of the beads exhibiting AChR clusters after only $2 \mathrm{~h}$. Moreover, the number of BARPs reached a plateau within $7 \mathrm{~h}$ of bead application. In contrast, infolding of sarcolemma at BARPs did not begin until 4-10 h after contact $(\square)$. The extent of infolding was measured as the percentage increase in the length of the sarcolemma profile at the bead over the length of the bead circumference in contact with the cell. The data on BARP development were obtained from light-microscopic observations. The data on infolding development were collected from electron micrographs. Each point was the mean of at least 20 bead-muscle contacts. The bars denote S.E.M.

cells at MTJs and NMJs in vivo, and at NARPs, hotspots (spontaneously formed receptor patches) and BARPs in vitro. We found that talin is absent from NMJs of tadpole myotomal and submaxillaris muscles, and from more than $40 \%$ of the NARPs. We therefore conclude that, at the NMJs we studied, talin is not necessary for maintaining AChR clusters that are induced by nerve.

The sites at which talin was consistently and highly concentrated were the MTJ, BARPs and ventral hotspots. These specializations are all sites of contact between the cell and a substratum. Furthermore, they share certain ultastructural features, including deep invaginations of the plasma membrane, a well-developed basal lamina, and the insertion of myofibrils into this membrane (this work; and Peng, 1987). Our electron micrographs of BARPs and myotomal MTJ/NMJ revealed that these invaginations occupy regions of the membrane that are distinct from the $\mathrm{AChR}$ cluster domain. A similar subdivision is evident at ventral $A C h R$ clusters, at which vinculin is associated with actin cables that are offset from the AChR cluster domains (Bloch \& Geiger, 1980; Bloch \& Pumplin, 1988). Not all ventral cell-substratum adhesion sites are also sites of AChR cluster formation. We believe, therefore, that the principal role of talin at the MTJ, BARPs, and ventral hotspots is to mediate cellsubstrate interactions and participate in the attachment of actin filaments at these regions.

The absence of elevated talin staining from myotomal and submaxillaris NMJs may be related to the absence of deep infoldings that separate receptor cluster domains at other NMJs. The NMJs of the rat diaphragm (Sealock et al. 1986) and of the Yenopus leg muscle (Fig. 3) are associated with deep infoldings and are stained with talin. Since talin appears at BARPs well before infolding development, it may be necessary for infolding development in these cells. Perhaps receptors are excluded from the infolding region as a result of the clustering of talin and other cytoskeleton-membrane linkage proteins associated with the infoldings.

Our studies in vitro revealed that talin colocalization with NARPs and dorsal hotspots was highly variable. Nearly half of the NARPs and $40 \%$ of dorsal hotspots were talin-free. Even at receptor clusters that exhibited a high degree of colocalization, there did not appear to be a one-to-one relationship between talin and AChRs. Since beads always induce talin-positive receptor clusters on the dorsal side of the muscle cells, we can eliminate the argument that the dorsal sides of these cells are less capable of concentrating talin. The variability we observed could be due to differences in signals that induce postsynaptic-type differentiation at these sites or to differences in the developmental states of the clusters. Regarding signals, our data are consistent with either one signal being sufficient to induce both types of differentiation or with two signals being provided by the stimulus, one for receptor clustering and one for cytoskeleton-membrane linkage. The ability of certain objects (e.g. beads) to induce colocalization recommends the one signal hypothesis, but the separation of these domains within the same cells (cf., submaxillaris NMJ and MTJ) recommends the two signal hypothesis. Regarding developmental states, our data on NARPs and dorsal hotspots are consistent either with talin arriving prior to receptors and subsequently dissociating from the receptor cluster, or with talin arriving after the appearance of receptor clusters. The variability we observed may indicate that more than one mechanism can be used to colocalize $\mathrm{AChR}$ clusters and talin accumulations.

The present work suggests that the postjunctional membrane is heterogeneous, containing a domain for $\mathrm{AChR}$ clusters and a domain for cytoskeletal proteins involved in cytoskeleton-membrane linkage. Although actin-associated proteins have been localized at NMJs, those results need to be re-evaluated at the ultrastructural level to determine in which domain these proteins are present. Only one peripheral membrane protein, the postsynaptic $43 \mathrm{~K}$ protein, has been localized to the receptor domain (Burden, 1985; Peng \& Froehner, 1985; Sealock et al. 1984). As demonstrated in this work, talin is associated with the cytoskeleton-membrane linkage domain. We suggest that talin is involved in the attachment of actin filaments and infolding formation in muscle cells, but it is not necessary for receptor clustering or cluster maintenance. The factors that stimulate the clustering of AChRs and the accumulation of talin at certain NMJs, as well as the mechanisms responsible for the organization of these domains, remain to be identified.

This work was supported by NIH grants NS-23583 (to H.B.P.) and GM-29860 (to K.B.), and by the Muscular Dystrophy Association grants (to H.B.P. and K.B.) and 
postdoctoral fellowship (to C.E.T.). We gratefully acknowledge the help of R. Sealock and K.-x. Gao in providing cryosections of Xenopus muscle.

\section{References}

ANDERSON, M. J. (1986). Nerve-induced remodeling of muscle basal lamina during synaptogenesis. $\mathcal{Y}$. Cell Biol. 102, 863-877.

Anderson, M. J. \& Cohen, M. W. (1977). Nerve-induced and spontaneous redistribution of acetylcholine receptors on cultured muscle cells. 7. Physiol. 268, 757-773.

Anderson, M. J. \& Fambrough, D. M. (1983). Aggregates of acetylcholine receptors are associated with plaques of a basal lamina heparan sulfate proteoglycan on the surface of skeletal muscle fibers. F. Cell Biol. 97, 1396-1411.

Bloch, R. J. \& Geiger, B. (1980). The localization of acetylcholine receptor clusters in areas of cell-substrate contact in cultures of rat myotubes. Cell 21, 23-35.

Bloch, R. J. \& HALL, Z. W. (1983). Cytoskeletal components of the vertebrate neuromuscular junction: vinculin, $\alpha$-actinin, and filamin. F. Cell Biol. 97, 217-223.

Bloch, R. J. \& Pumplin, D. W. (1988). Molecular events in synaptogenesis: nerve-muscle adhesion and postsynaptic differentiation. Am. Y. Physiol. 254, C345-C364.

Buck, C. A. \& Horwitz, A. F. (1987). Cell surface receptors for extracellular matrix molecules. A. Rev. Cell Biol. 3, 179-205.

BURden, S. J. (1985). The subsynaptic $43 \mathrm{kDa}$ protein is concentrated at developing nerve-muscle synapses in vitro. Proc. natn. Acad. Sci. U.S.A. 82, 8270-8273.

Burden, S. J., Sargent, P. B. \& McMahan, U. J. (1979). Acetylcholine receptors in regenerating muscle accumulate at original synaptic sites in the absence of the nerve. 7 . Cell Biol. 82 , $412-425$.

BuRRIDGE, K. (1986). Substrate adhesions in normal and transformed fibroblasts: organization and regulation of cytoskeletal, membrane and extracellular matrix components at focal contacts. Cancer Rev. 4, 18-78.

Burridge, K. \& ConNell, L. (1983). A new protein of adhesion plaques and ruffling membranes. F. Cell Biol. 97, 359-367.

Burridge, K. \& Mangeat, P. (1984). An interaction between vinculin and talin. Nature, Lond. 308, 744-746.

Chow, I. \& COHEN, M. W. (1983). Developmental changes in the distribution of acetylcholine receptors in the myotomes of Xenopus laevis. J. Physiol. 339, 553-571.

Feramisco, J. R. \& Burridge, K. (1980). A rapid purification of $\alpha$ actinin, filamin and a 130,000 dalton protein from smooth muscle. J. biol. Chem. 255, 1194-1199.

Fraker, P. J. \& Speck, J. C. (1978). Protein and cell membrane iodinations with a sparingly soluble chloramine $1,3,4,6$-tetrachloro3 alpha, 6 alpha diphenylglycouril. Biochem. biophys. Res. Commun. 80, 849-857.

Hall, Z. W., Lubit, B. W. \& Schwartz, J. H. (1981). Cytoplasmic actin in postsynaptic structures at the neuromuscular junction. J. Cell Biol. 90, 789-792.

Henderson, L. P., Smith, M. A. \& SpItzer, N. C. (1984). The absence of calcium blocks impulse-evoked release of acetylcholine but not de novo formation of functional neuromuscular synaptic contacts in culture. $\mathcal{F}$. Neurosci. 4, 3140-3150.

Hunter, W. M. \& Greenwood, F. C. (1962). Preparation of iodine131 labeled human growth hormone of specific activity. Nature, Lond. 194, 495-496.

LAEMMLI, U. K. (1970). Cleavage of structural proteins during the assembly of the head of bacteriophage T4. Nature, Lond. 227, $680-685$.

Moody-CorbetT, F. \& COHEN, M. W. (1982). Increased adhesiveness at sites of high acetylcholine receptor density on embryonic amphibian muscle cells cultured without nerve. 7. Embryol. exp. Morph. 72, 53-69.

Nitkin, R. M., Smith, M. A., Magill, C., Fallon, J. R., Yao, Y. M., Wallace, B. G. \& MCMaHAN, U. J. (1987). Identification of agrin, a synaptic organizing protein from torpedo electric organ. y. Cell Biol. 105, 2471-2478.

O'Halloran, T. \& Burridge, K. (1986). Purification of a $190 \mathrm{kDa}$ protein from smooth muscle: relationship to talin. Biochim. biophys. Acta 869, 337-349.

PENG, H. B. (1987). Development of the neuromuscular junction in tissue culture. CRC Crit. Rev. anat. Sci. 1, 91-131.

Peng, H. B., Chen, Q., Rochlin, W., Tobler, M., Turner, C. E \& Burridge, K. (1987). The distribution of talin at $\mathrm{ACh}$ receptor clusters in muscle cells. 7 . Cell Biol. 105, 63a

Peng, H. B. \& Cheng, P.-C. (1982). Formation of postsynaptic specializations induced by latex beads in cultured muscle cells. F. Neurosci. 2, 1760-1774

Peng, H. B. \& Froehner, S. C. (1985). Association of the postsynaptic $43 \mathrm{~K}$ protein with newly formed acetylcholine receptor clusters in cultured muscle cells. $\mathcal{~ f . ~ C e l l ~ B i o l . ~ 1 0 0 , ~ 1 6 9 8 - 1 7 0 5 . ~}$

Peng, H. B. \& NaKajima, Y. (1978). Membrane particle aggregates in innervated and noninnervated cultures of Xenopus embryonic muscle cells. Proc. natn. Acad. Sci. U.S.A. 75, 500-504.

Peng, H. B. \& Phelan, K. A. (1984). Early cytoplasmic specialization at the presumptive acetylcholine receptor cluster: a meshwork of thin filaments. F. Cell Biol. 99, 344-349.

Sanes, J. R., Schachner, M. \& Covault, J. (1986). Expression of several adhesive macromolecules (N-CAM, L1, J1, NILE, uvomorulin, laminin, fibronectin, and a heparan sulfate proteoglycan) in embryonic, adult, and denervated adult skeletal muscle. $\mathscr{~}$. Cell Biol. 102, 420-431.

Sealock, R., Paschal, B., Beckerlie, M. \& Burridge, K. (1986). Talin is a post-synaptic component of the rat neuromuscular junction. Expl Cell Res. 163, 143-150.

Sealock, R., Wray, B. E. \& Froehner, S. C. (1984). Ultrastructural localization of the $M_{\mathrm{r}} 43,000$ protein and the acetylcholine receptor in Torpedo postsynaptic membranes using monoclonal antibodies. F. Cell Biol. 98, 2239-2244.

SHEAR, C. R. \& BLOCH, R. J. (1985). Vinculin in subsarcolemmal densities in chicken skeletal muscle: localization and relationship to intracellular and extracellular structures. $\mathcal{Y}$. Cell Biol. 101, 240-256.

Tidrall, J. G., O'Halloran, T. \& Burridge, K. (1986). Talin at myotendinous junctions. F. Cell Biol. 103, 1465-1472.

Towbin, H., Staehelin, T. \& Gordon, J. (1979). Electrophoretic transfer of proteins from polyacrylamide gels to nitrocellulose sheets: procedure and some applications. Proc. natn. Acad. Sci. U.S.A. 76, 4350-4354.

Trotter, J. A., Corbett, K. \& Avner, B. P. (1981). Structure and function of the murine muscle-tendon junction. Anat. Rec. 201, 293-302.

Trotter, J. A., Eberhard, S. \& Samora, A. (1983). Structural connections of the muscle-tendon junctions. Cell Motil. 3, 431-438.

WachsSTOCK, D. H., Wilkins, J. A. \& Lin, S. (1987). Specific interaction of vinculin and $\alpha$-actinin. Biochem. biophys. Res. Commun. 146, 554-560.

Weldon, P. R. \& Cohen, M. W. (1979). Development of synaptic ultrastructure at neuromuscular contacts in an amphibian cell culture system. I. Neurocytol. 8, 239-259.

Wilkins, J. A., Chen, K. Y. \& LiN, S. (1983). Detection of high molecular weight vinculin binding proteins in muscle and nonmuscle tissues with an electroblot-overlay technique. Biochem. biophys. Res. Commun. 116, 1026-1032.

(Received 29 August 1988 - Accepted, in revised form, 2 December 1988 